\title{
Single-Atom Scale Structural Selectivity in Te Nanowires Encapsulated Inside Ultranarrow, Single-Walled Carbon Nanotubes
}

Paulo V. C. Medeiros, ${ }^{*}{ }^{\dagger} \odot$ Samuel Marks, ${ }^{\ddagger}$ Jamie M. Wynn, ${ }^{\dagger}$ Andrij Vasylenko, ${ }^{\ddagger}, I \odot$ Quentin M. Ramasse, ${ }^{\S}$ David Quigley, ${ }^{\ddagger}, \|$ Jeremy Sloan, ${ }^{*}, *$ and Andrew J. Morris ${ }^{*,+, \dagger}$

${ }^{\dagger}$ Theory of Condensed Matter Group, Cavendish Laboratory, University of Cambridge, J. J. Thomson Avenue, Cambridge CB3 0HE, United Kingdom

${ }^{\ddagger}$ Department of Physics and "Centre for Scientific Computing, University of Warwick, Coventry CV4 7AL, United Kingdom

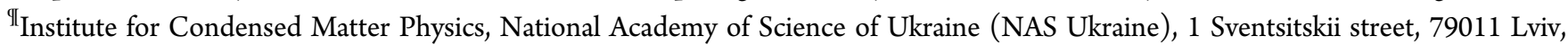
Ukraine

${ }^{\S}$ SuperSTEM Laboratory, SciTech Daresbury Campus, Keckwick Lane, Daresbury WA44AD, United Kingdom

Supporting Information

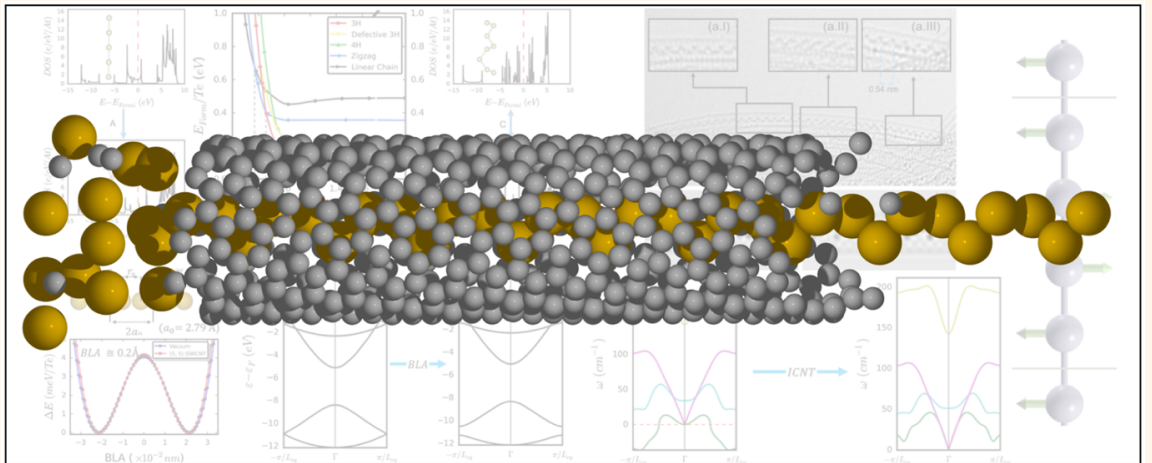

ABSTRACT: Extreme nanowires (ENs) represent the ultimate class of crystals: They are the smallest possible periodic materials. With atom-wide motifs repeated in one dimension (1D), they offer a privileged perspective into the physics and chemistry of low-dimensional systems. Single-walled carbon nanotubes (SWCNTs) provide ideal environments for the creation of such materials. Here we present a comprehensive study of Te ENs encapsulated inside ultranarrow SWCNTs with diameters between $0.7 \mathrm{~nm}$ and $1.1 \mathrm{~nm}$. We combine state-of-the-art imaging techniques and 1D-adapted ab initio structure prediction to treat both confinement and periodicity effects. The studied Te ENs adopt a variety of structures, exhibiting a true 1D realization of a Peierls structural distortion and transition from metallic to insulating behavior as a function of encapsulating diameter. We analyze the mechanical stability of the encapsulated ENs and show that nanoconfinement is not only a useful means to produce ENs but also may actually be necessary, in some cases, to prevent them from disintegrating. The ability to control functional properties of these ENs with confinement has numerous applications in future device technologies, and we anticipate that our study will set the basic paradigm to be adopted in the characterization and understanding of such systems.

KEYWORDS: extreme nanowires, carbon nanotubes, encapsulation, 1D-AIRSS, mismatch, implicit nanotubes, nanoconfinement

$\mathrm{E}$ xtreme nanowires $(\mathrm{ENs})^{1}$ are few-atom-wide wires that extend along one dimension and have their fine structural details resolved also at the atomic length scale. They represent the smallest possible one-dimensional (1D) materials; a fact which, on its own, justifies detailed experimental and theoretical investigation into materials structure and characterization. In contrast to many other exotic materials predicted via computational discovery, practical routes to EN synthesis have revealed a rich landscape of $1 \mathrm{D}$ physics and chemistry.
Such extreme 1D structures have an obvious potential for use as interconnecting elements between components in nanocircuits, but less immediate applications, such as the design of nanophasechange materials (NPCMs) for use in high-efficiency solid-state storage devices, can be envisioned, especially since structural

Received: March 30, 2017

Accepted: $\quad$ May 3, 2017

Published: May 3, 2017 
changes in 1D can be induced-and precisely characterized, as we show here-by the equally extreme confinement of matter at the nanoscale.

A practical route to achieving nanoconfinement in $1 \mathrm{D}$ is making use of single-walled carbon nanotubes (SWCNTs). A prominent characteristic of SWCNTs is their capillarity, ${ }^{2}$ which has been explored in experiments to grow and encapsulate crystals, molecules, and aggregates. SWCNTs have remarkable chemical stability and provide a very effective chemical shielding from the external environment. These characteristics make such tubes the ideal templates for the discovery of nanomaterials as well as for the investigation of new material phases that emerge due to nano-1D confinement. ${ }^{3-7}$ Conversely, we have recently shown that encapsulated ENs can be used to boost electronic transport in SWCNTs. ${ }^{8}$ This further highlights the importance of such materials for applications in electronics, especially with the demonstrated possibility of building SWCNT-based transistors with gate lengths as small as $1 \mathrm{~nm}$. ${ }^{9}$ Hybrid nanotube-nanowire systems have been used in the fabrication of nanothermometers ${ }^{10}$ and magnetic force microscopy sensors, ${ }^{11}$ and potential for many other applications, ${ }^{12}$ such as battery electrodes ${ }^{13}$ and in photothermal nanomaterials-based devices, ${ }^{14}$ has also been demonstrated.

Applications of alloys containing $\mathrm{Ge}, \mathrm{Sb}$, and $\mathrm{Te}$ in phasechange materials have enjoyed considerable success over the past decades, ${ }^{15}$ and the search for NPCMs based on such elements is a natural step. ${ }^{16}$ Te is of particular interest in this context because its bulk structure favors the formation of $1 \mathrm{D}$ structures. ${ }^{17,18}$ Ultranarrow Te nanowires down to $25 \mathrm{~nm}$ in diameter exhibit a wealth of interesting properties related to their photoconductivity, nonlinear optical response, thermoelectric, and piezoelectric effects. $^{19,20}$ They have been employed in gas sensors, ${ }^{21}$ optoelectronic devices, ${ }^{22}$ and photonic crystals. ${ }^{23}$ Recently, the dimensionality of Te wires was reduced to single-atom chains in carbon nanotubes (CNTs) with internal diameters in the range $1.2 \pm 0.2 \mathrm{~nm}^{24}$ There is, however, a clear motivation to produce a more consistent, atomically regulated material, ideally encapsulated exclusively within single conformation ultranarrow single-walled carbon nanotubes (UNSWCNTs) with diameters smaller than $1.2 \mathrm{~nm}$. Such UNSWCNTs would have precisely tailored physical properties, being either pure semimetallic or pure semiconducting confining tubes with a single discrete gap.

Recent major developments in hardware for aberration corrected high-resolution transmission electron microscopy (AC-TEM) and aberration corrected scanning transmission electron microscopy (AC-STEM) $)^{25,26}$ have driven considerable interest in low-dimensional materials, as imaging such systems requires very high precision. Such advancements, on the other hand, mean that three-dimensional data with atomic-dimension accuracy are now needed for ENs if one is to ensure maximum compatibility between theory and experiment. From the theory standpoint, such developments are paralleled by the advent of high-performance computing, which, allied with clever approaches to structure searching, can increase the efficiency and accuracy in materials discovery and eliminate guesswork from the process.

Here, we combine AC-TEM, AC-STEM, and electron energy loss spectroscopy (EELS) measurements with a high-throughput, $1 \mathrm{D}$-adapted implementation of the $a b$ initio random structure searching (AIRSS) method ${ }^{27}$ to provide the most accurate characterization to date of Te ENs grown inside UNSWCNTs. The AIRSS methodology has successfully been employed to tackle problems as diverse as the structural determination of point-defects, ${ }^{28-30}$ prediction of high-pressure phases, ${ }^{31}$ as well as to detect structural transformations in nanostructured siliconbased lithium-ion batteries. ${ }^{32}$

\section{RESULTS AND DISCUSSION}

We have filled UNSWCNTs with diameters spanning from $0.7 \mathrm{~nm}$ to $1.1 \mathrm{~nm}$ with Te nanowires using a similar sublimation

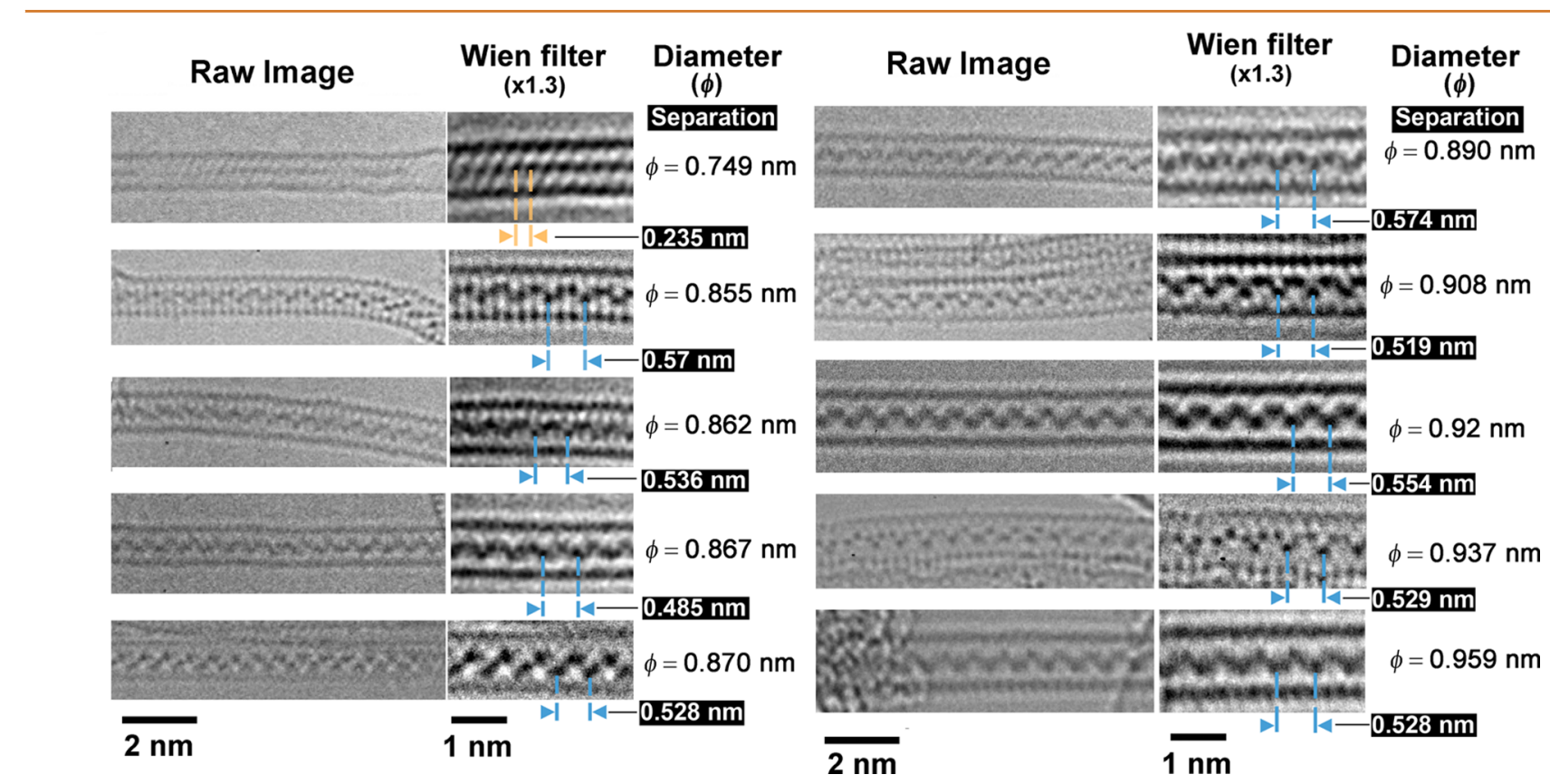

Figure 1. AC-TEM images of UNSWCNTs, sorted by diameter (smallest to largest), filled with 1D Te extreme nanowires ENs. With the exception of the LC encapsulated inside the smallest diameter $(0.749 \mathrm{~nm})$ UNSWCNT (Te-Te spacing indicated in orange), all of the remaining ENs form coil structures with differing periods (coil pitches) along the lengths of each nanotube. UNSWCNT diameters and Te atomic period measurements were obtained from the indicated Wien filtered sections with an estimated precision of $\pm 0.008 \mathrm{~nm}$. 


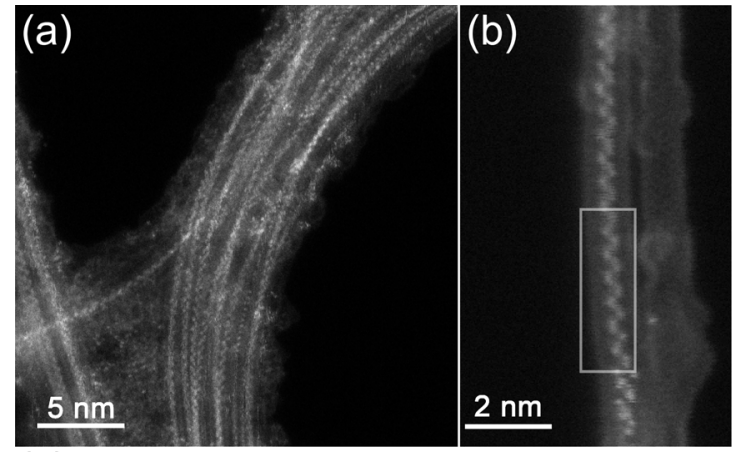

(c)

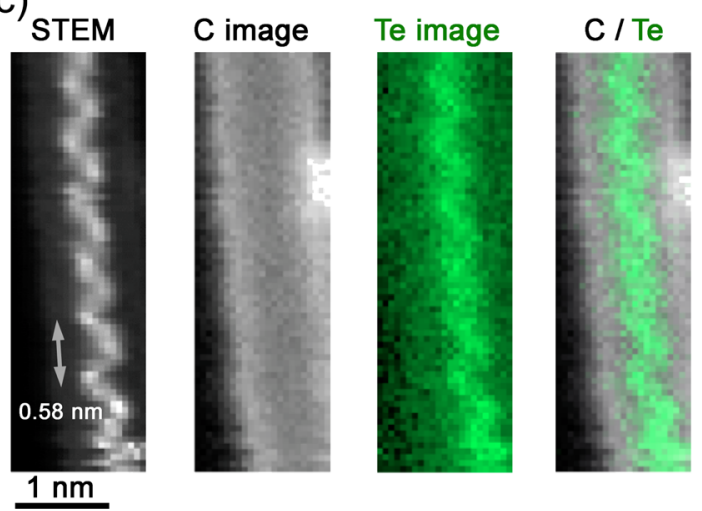

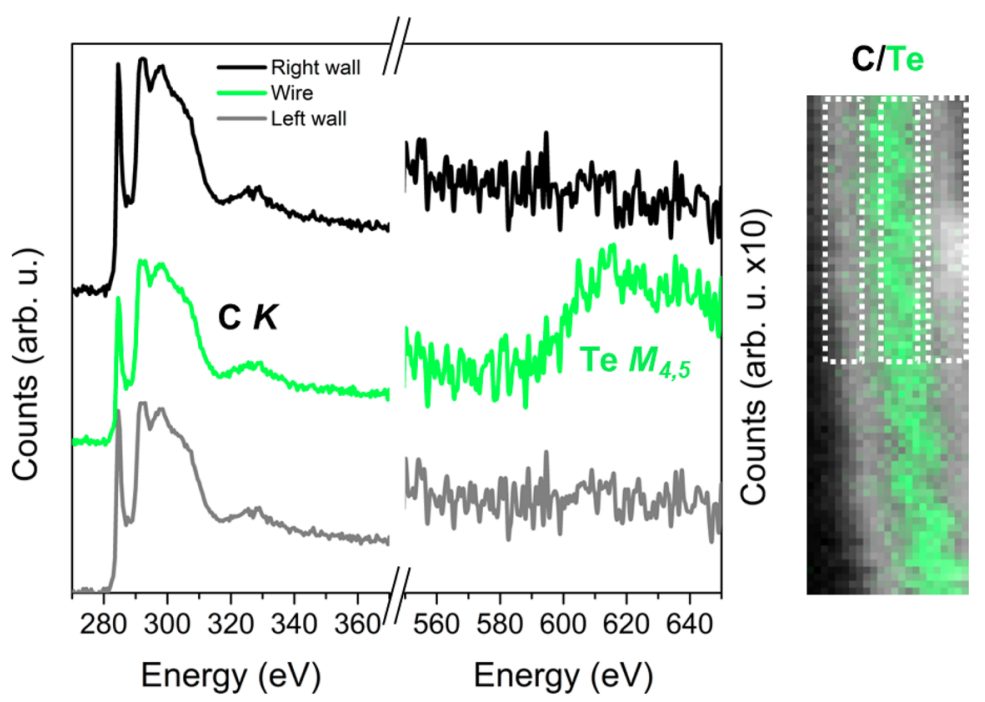

Figure 2. STEM and EELS. (a) Medium magnification STEM ADF image of narrow bundles of UNSWCNTs filled with Te 3H ENs. Filling percentage of UNSWCNTs was determined to be $c a .70 \%$. (b) Higher magnification STEM ADF image showing two UNSWCNTs, one filled with a Te coil (left) and one empty. The boxed region was used for the spectrum imaging recorded in (c) and the EELS spectrum recorded in (d). (c) STEM image, $\mathrm{C}$, Te and combined $\mathrm{C} / \mathrm{Te}$ spectrum images recorded from the boxed region in (b), confirming the chemical identity of the Te coil. (d) C K edge EELS spectrum and Te M 4,5 edge EELS spectra recorded from the regions indicated in the corresponding detail (inset, right). We found no indication in our EELS measurements that oxygen is present in the immediate vicinity of the wires.

method to that applied by Senga et al. ${ }^{33}$ to the filling of doublewalled CNTs with ionic CsI single-atom chains. Elemental Te was filled by sublimation in vacuo (see Experimental Methods section) directly into the UNSWCNTs to produce single-atomthick Te ENs. Our AC-TEM imaging results (Figure 1) show that the vast majority of the obtained Te ENs consisted of singleatom-thick Te 3-fold-symmetry helical coils (3Hs). We found that the "pitch" of each coil varies in a nonmonotonic fashion with respect to the observed diameter of the encapsulating UNSWCNT. See the included Supporting Information (SI) for more details. Scanning TEM (STEM) imaging and EELS (Figure $2 a-d$ ) confirmed the chemical identity of the obtained Te coils as elemental Te.

In order to unambiguously determine the structures of the encapsulated Te ENs, we adapted the AIRSS method ${ }^{27}$ to the modeling of SWCNT-encapsulated ID structures (see SI). Additionally, we note that the determination of the most favorable EN structure encapsulated inside a single SWCNT is not enough to provide a precise characterization of SWCNT-encapsulated ENs. The formation of distinct EN structures can be favored by modifying the diameter of the encapsulating SWCNT, and the ability to predict the most favorable structures inside SWCNTs of any given diameter is thus paramount. Particularly at the very small length scale characteristic of confinement inside UNSWCNTs, drastic changes in the shapes of the encapsulated ENs are expected to occur at certain critical UNSWCNT diameters, and a map of such structural changes can be drawn by following the best diameter-dependent encapsulated structures. We have thus selected the most energetically favorable structures obtained from our AIRSS screening on Te ENs and produced the diagram shown in Figure 3. As discussed in the SI, to deal with strain and mismatch between the ENs and the encapsulating UNSWCNTs, the computational procedure to obtain such a diagram involves the use of implicit SWCNTs (ICNTs). We discuss the ICNT model in the SI.

We can assert by analyzing the diagram in Figure 3 that the only viable geometry for the Te EN encapsulated inside UNSWCNT with diameters below $0.77 \mathrm{~nm}$ is that of a linear chain (LC). The threshold of $0.77 \mathrm{~nm}$ for the emergence of the LC structure is compatible with the van der Waals (vdW) radii of $\mathrm{C}$ and $\mathrm{Te}$, which are approximately $0.17 \mathrm{~nm}$ and $0.21 \mathrm{~nm}$, respectively. Repulsion between the electronic clouds of the encapsulated Te atoms and the UNSWCNT walls increases rapidly for diameters below the calculated threshold, and the LC configuration is the one that minimizes such an overlap. Due to interactions between electron beam and the imaged specimens, we could not ascertain the existence of a Peierls distortion (PD $)^{35}$ directly from our experimental data. In our simulations, however, we establish that such distortion is required for the encapsulated Te LC to be mechanically stable and, therefore, observable (see Figure 4). The calculated bond length alternation (BLA) is of approximately $0.02 \mathrm{~nm}$, leading to a reduction of about $4 \mathrm{meV} / \mathrm{Te}$ in the total energy of the chain. This BLA does not vary significantly with the diameter of the encapsulating SWCNT. The characteristic effect of a PD in the electronic structure of a LC is the lifting of band degeneracies at the boundaries of the Brillouin zone (of the new two-atom primitive unit cell (PC)). This is also illustrated in Figure 4 for the present case. Note, however, that the Te LC remains metallic 

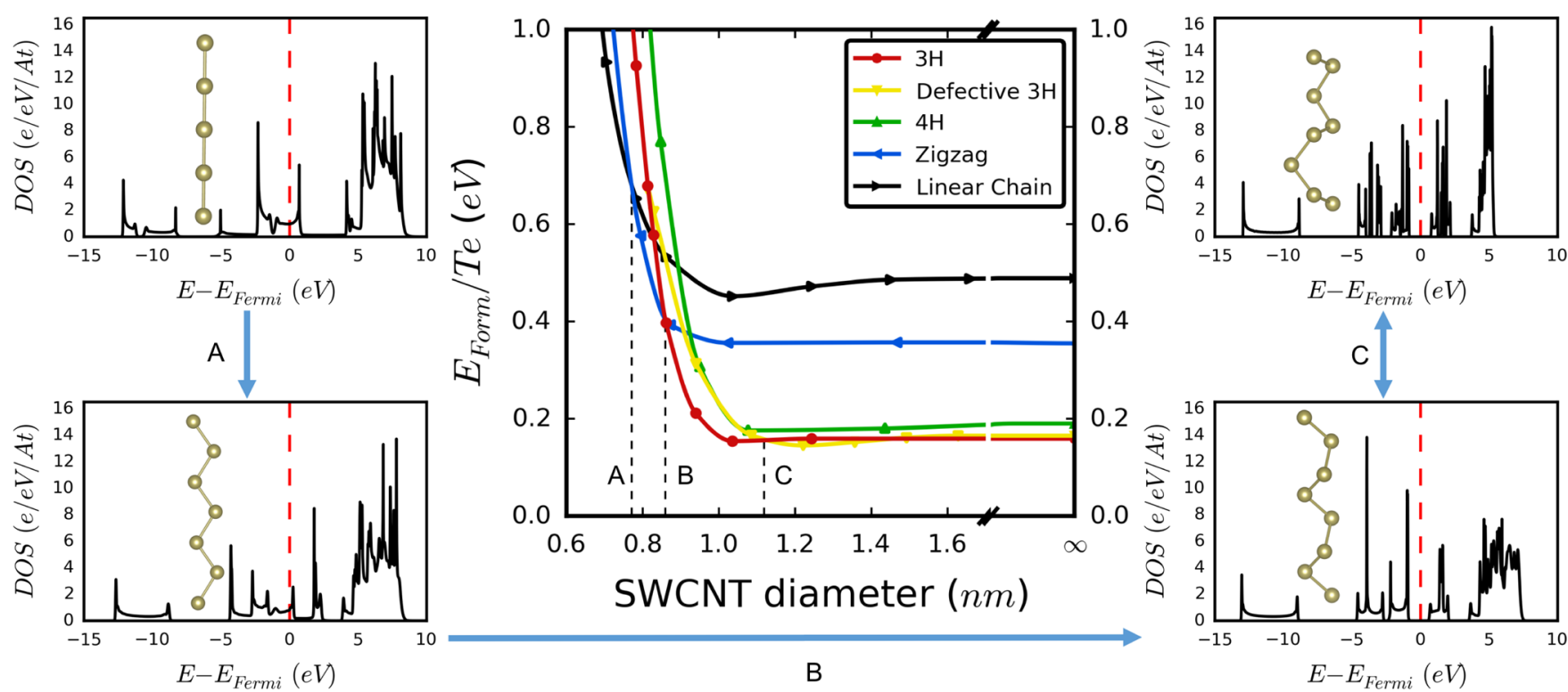

Figure 3. Competing structures of SWCNT-encapsulated Te extreme nanowires. Central panel: Formation energy per encapsulated atom as a function of the encapsulating diameter. The labels A-C mark the diameters at which we predict structural transitions to occur. Left and right panels: Geometries of the structures involved in the transitions A-C, along with the corresponding densities of states (DOS). The competing structures of the ENs were determined using the AIRSS method. The DOS were calculated using the OptaDOS code. ${ }^{34}$
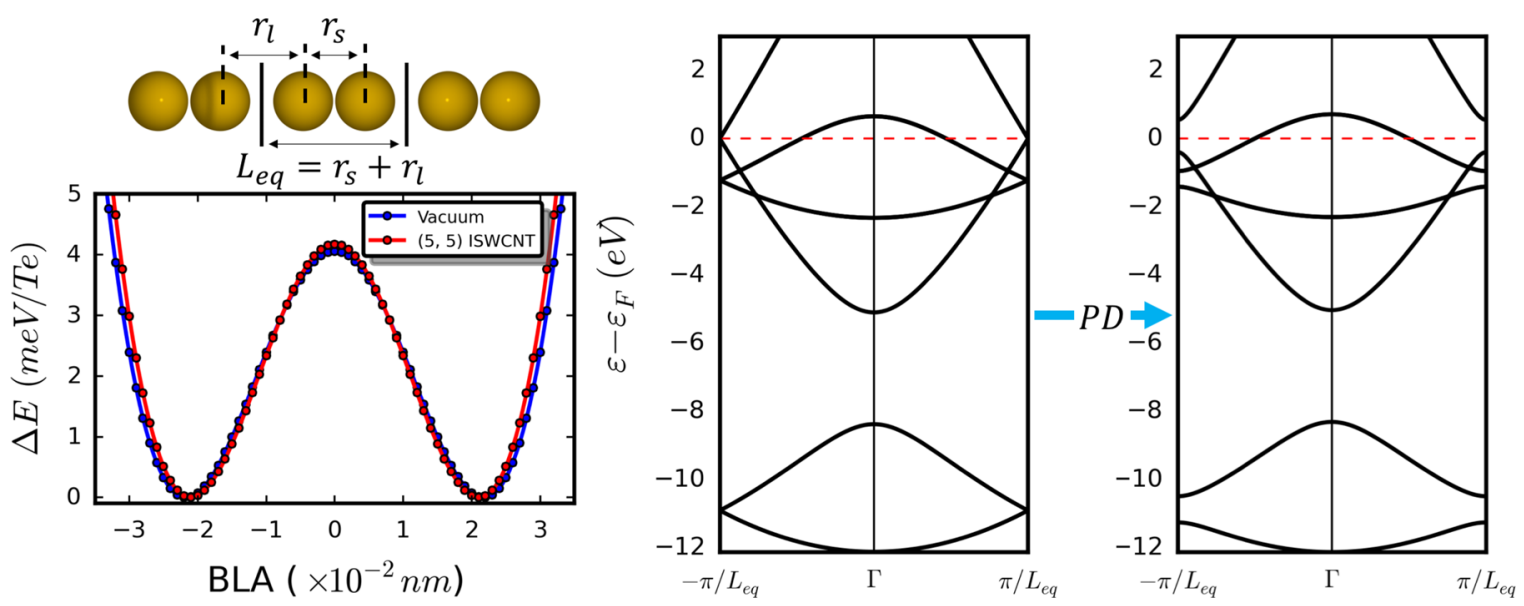

Figure 4. Total energy shifts ( $\Delta E$ vs BLA for the Te LC: Chain in vacuum and encapsulated inside a $(5,5)$ ICNT, with BLA $\equiv r_{l}-r_{s}$. In vacuum, the optimal BLA is $0.022 \mathrm{~nm}$, and the excess energy for the BLA = 0 case is $4.06 \mathrm{meV} / \mathrm{Te}$. For the encapsulated LC, the corresponding values are $0.021 \mathrm{~nm}$ and $4.17 \mathrm{meV} /$ Te. Electronic band structures: Left-hand side: Chain without PD (i.e., BLA = 0). Right-hand side: PD chain with

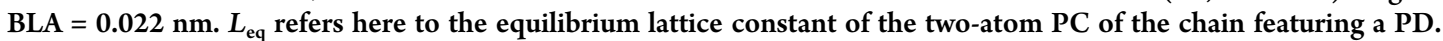

despite the presence of the $\mathrm{PD}$, as one of the electronic bands continues to cross the Fermi level. Given that PDs are almost exclusively associated with metallic-semimetallic electronic transitions in the literature, the metallic behavior we predict for the encapsulated Te LC might seem contradictory at first. We point out, nonetheless, that atomic chains exhibiting metallic behavior albeit featuring PDs have previously been predicted in different contexts. ${ }^{36,37}$ We have also found that the Te LC cannot form in vacuum, as it has an unstable transverse acoustic vibrational mode. Such an instability, however, is quenched upon encapsulation, making the encapsulated chain stable. The calculated phonon spectra for the chain in both cases is shown in Figure 5a. The Te LC is therefore an example of an EN for which nanoconfinement is not only a sufficient condition for mechanical stability but also a necessary one.

We predict the first Te EN structural transition to occur in the vicinity of $d=0.77 \mathrm{~nm}$ (labeled A in Figure 3), shortly followed by another one at $d \approx 0.86 \mathrm{~nm}$ (marked $\mathrm{B})$. From $\mathrm{A}$, our calculations indicate that the zigzag $(\mathrm{ZZ})$ chain configuration becomes accessible for the encapsulated Te ENs, remaining so until the diameters of the encapsulating UNSWCNT reach approximately $0.86 \mathrm{~nm}$. With $n$ and $m$ defined as discussed in the SI, we can narrow the possible UNSWCNTs possessing diameters in the A-B interval down to $(8,3),(10,0),(9,2),(6,6),(7,5)$, $(10,1),(8,4)$, and $(9,3)$, in order of increasing diameters. The choice $m \geq 0$ (right-hand chirality SWCNTs) was made without loss of generality, as we verified that the chirality of the encapsulating UNSWCNTs does not affect the energetics of the encapsulated systems. As in the case of the Te LC, we found that the Te $\mathrm{ZZ}$ chain is mechanically unstable in vacuum and therefore cannot form in such an environment. Once more, however, the chain is stabilized upon encapsulation, as shown in Figure $5 b$. Such a chain therefore provides another example of ENs that require nanoconfinement to achieve mechanical stability. 

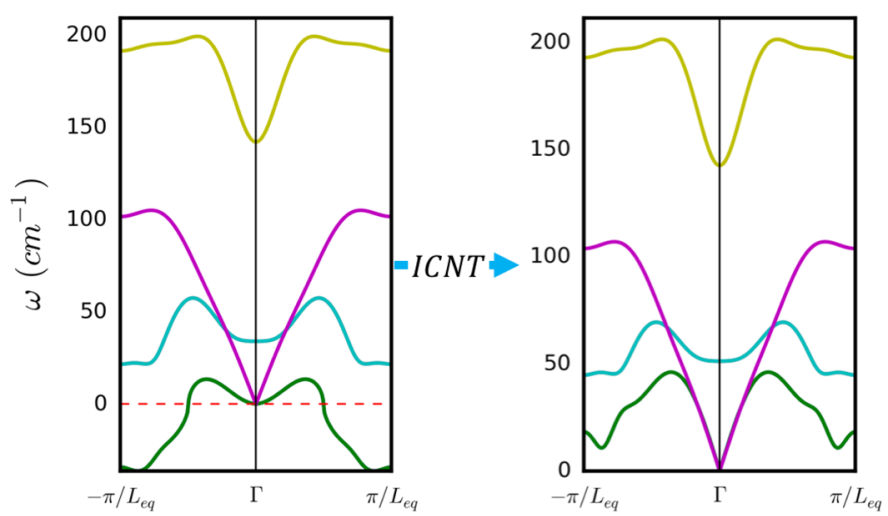

(a)
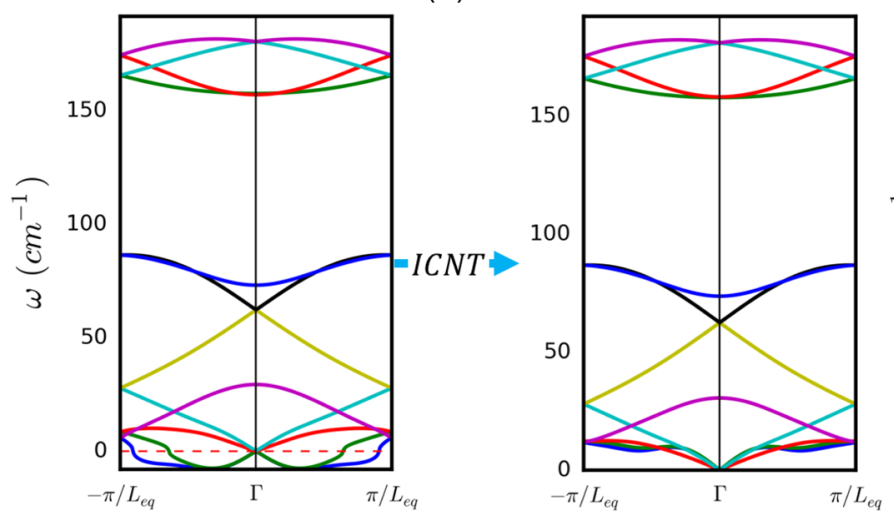

(c)
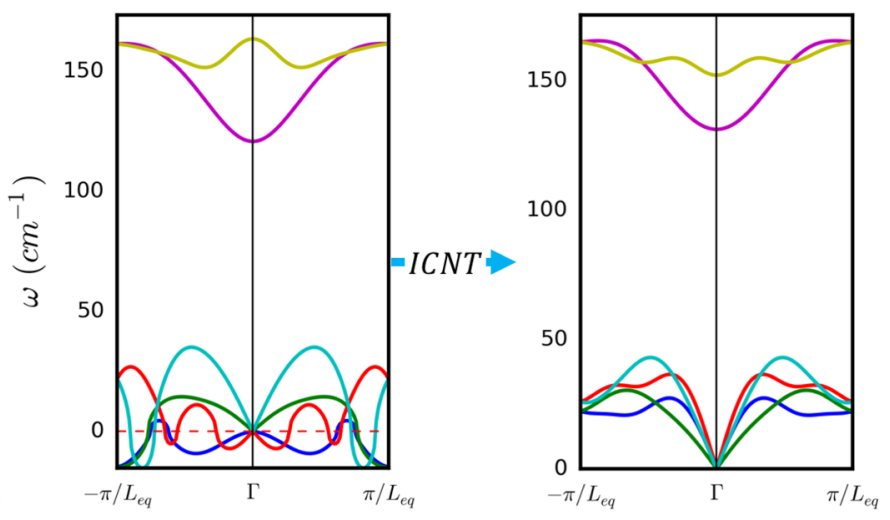

(b)
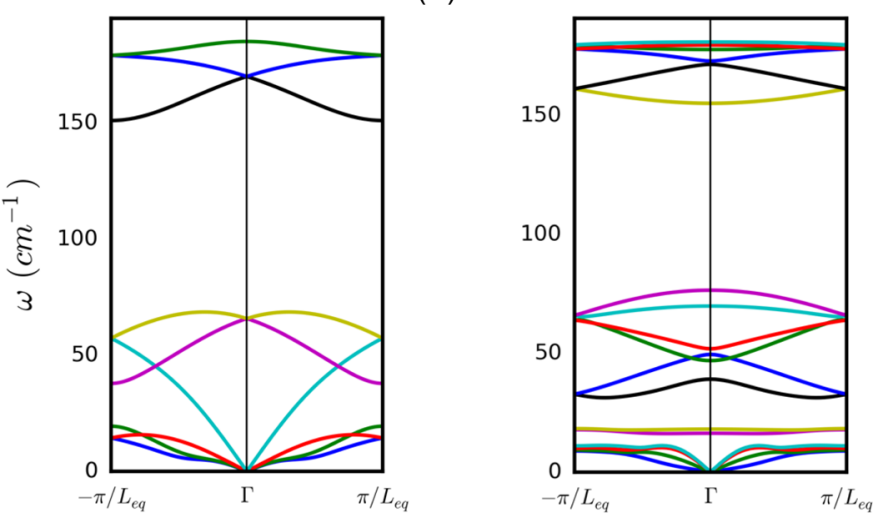

(d)

Figure 5. Phonon spectra for the (a) LC, (b) $\mathrm{ZZ},(\mathrm{c}) 4 \mathrm{H}$, and (d) $3 \mathrm{H}$ chains. The figures in (a-c) show the spectra calculated for the bare chains (left) and for the chains encapsulated inside $(5,5),(8,3)$, and $(11,5)$ ICNTs, respectively (right). The unstable acoustic modes in vacuum are stabilized upon encapsulation. In (d), the vacuum spectra calculated for the ideal (left) and defective (right) $3 \mathrm{H}$ chains are presented. Both $3 \mathrm{H}$ chains are mechanically stable.

From B, we predict the $\mathrm{ZZ}$ configuration to become less likely to occur, and the $3 \mathrm{H}$ form of $\mathrm{Te},{ }^{38}$ the $1 \mathrm{D}$ building block of the bulk phase of the element, becomes the dominant structure. Depending on whether this $3 \mathrm{H}$ building block has right- or lefthand screw symmetry, bulk Te belongs to space groups 152 or 154 , respectively. We have found that both chiral orientations of the $3 \mathrm{H}$ structure have practically identical formation energies and are thus equally likely to form inside UNSWCNTs. Interestingly, we found that a defective version of the $3 \mathrm{H}$ structure becomes energetically competitive for SWCNT diameters starting from $\sim 1.1 \mathrm{~nm}$ (marked C in Figure 3). Such a structure can be seen as a combination of two intercalated ideal $3 \mathrm{H}$ chains: one possessing left- and another right-handed screw symmetry. Both the ideal and defective $3 \mathrm{H}$ chains are mechanically stable, as can be seen from their phonon spectra presented in Figure $5 \mathrm{~d}$. The fact that defective forms of the $3 \mathrm{H}$ EN are energetically favorable compared with the ideal configuration indicates the possibility of triggering structural changes, through the use of external stimuli, to displace Te atoms from their ideal positions. This could be performed, for instance, by irradiation with high-energy electron beams, although one has to exercise care in doing this, as we found in our experiments that the Te chains can become highly mobile, or even unstable, when interacting with high-energy electron beam radiation. Such a high mobility even caused the encapsulating SWCNTs to become empty again on a few occasions. Finally, we point out that, given the proximity of the curves representing the $3 \mathrm{H}$ and $\mathrm{ZZ}$ structures in Figure 3, such structures can reasonably be expected to coexist at the range of diameters between $\mathrm{A}$ and $\mathrm{B}$.

In the left- and right-hand side panels in Figure 3 we present the electronic densities of states for the Te structures involved in the transitions $\mathrm{A}-\mathrm{C}$. Owing to the general chemical inertness of SWCNTs and to the particular tendency shown by Te to form covalent intrachain bonds and only weak, vdW-mediated interchain connections, the effects of EN-SWCNT interactions on the electronic structure of the encapsulated ENs are small. By inspecting the plots, we conclude that the structural change marked $\mathrm{B}$ also represents a transition from a metallic to a semiconducting EN state. Since encapsulating ENs inside SWCNTs is analogous to submitting the ENs to external pressure, the possibility demonstrated here of inducing metalsemiconductor transitions by encapsulation of Te ENs inside UNSWCNTs means that encapsulation can provide a controllable route toward the construction of pressure-stabilized on-off switches based on Te ENs, despite the semiconducting nature of bulk Te.

Apart from the ground-state structures discussed so far, our AIRSS screening also yielded a number of metastable structures. From such structures, we selected those whose curves in the structure diagram (Figure 3 ) that are located sufficiently close, at least at one point, to the curves corresponding to the diameterdependent ground-state geometries. We consider energies to be sufficiently close when they are separated by about $1 k_{\mathrm{B}} T$, where $k_{\mathrm{B}}$ is the Boltzmann constant and $T$ represents the standard room temperature. At such a temperature, $k_{\mathrm{B}} T \approx 25.7 \mathrm{meV}$. One of 
such metastable structures is a double LC (two parallel LCs), which we found to be energetically accessible at diameters around $0.85 \mathrm{~nm}$. Upon further inspection, however, we found that the double LC has a mechanically unstable longitudinal acoustic vibrational mode that cannot be stabilized by encapsulation. For this reason, we have not included this structure in our diagram. We verified that such a mode does not represent a Peierls instability. The other metastable structure is a four-fold-symmetry helix $(4 \mathrm{H})$ akin to the one previously suggested for encapsulated sulfur. ${ }^{39}$ Although we have not observed such a structure in our experiments, we have determined that it is mechanically stable upon encapsulation (see Figure 5c) and could thus be accessible in small fragments. Our structure diagram indicates that this could occur for encapsulating SWCNTs diameters around $1.1 \mathrm{~nm}$ - the upper limit for the SWCNT diameters we considered experimentally.

Figure 6 presents a comparison between our experimental and simulated TEM images for the encapsulated Te $3 \mathrm{H}$ EN.

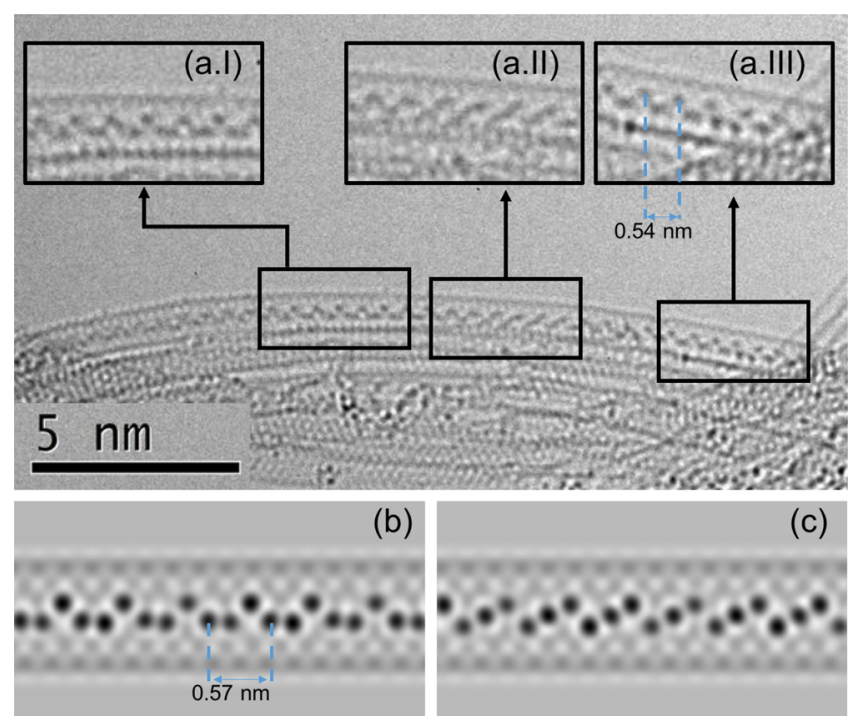

Figure 6. (a) Experimental and (b, c) simulated TEM images for encapsulated Te $3 H$. The SWCNT diameter in (a) is $0.945 \pm 0.015 \mathrm{~nm}$. The simulated images correspond to the most energetically favorable structure found here using AIRSS for Te encapsulated inside an explicit $(7,7)$ SWCNT $(d=0.949 \mathrm{~nm})$. They differ only by a rotation around the common translation axis. We found that the energy required to compress the AIRSS structure so that theoretical and experimental coil pitches coincide is about $20 \mathrm{meV} / \mathrm{Te}$. This is $<1 k_{\mathrm{B}} T / \mathrm{Te}$ at the adopted experimental conditions. The agreement between the geometries shown in (a.I), (a.III), and (b), as well as between (a.II) and (c), is excellent. Simulated images produced using the SimulaTEM code. ${ }^{40}$

The model in the simulated images corresponds to the most energetically favorable structure we predict for an encapsulating SWCNT with a diameter of $0.949 \mathrm{~nm}$, while the experimental TEM image was obtained for encapsulated Te inside a SWCNT with an estimated diameter of $0.945 \pm 0.015 \mathrm{~nm}$. Images (a.I) and (a.III) clearly represent domains inside the encapsulating SWCNT in which the structure shown in Figure $6 \mathrm{~b}$ has been formed. Most interestingly, however, is the observation that, although the structure in image (a.II) looks distinct from (a.I) and (a.III), and could thus, in principle, correspond to a different EN structure, it matches exactly the simulated image (c), which has been obtained using the same model as in (b), differing only by a rotation around the translation axis. We can therefore affirm that (a.II) also corresponds to an encapsulated Te $3 \mathrm{H}$ EN The very low frequencies (Figure $5 \mathrm{~d}$ ) of both the ideal and defective $3 \mathrm{H}$ acoustic torsional vibration modes (modes that correspond to a rigid rotation of the entire system around its symmetry axis at the limit $q \rightarrow 0$ ) indicate that the energy cost to twist such structures around their symmetry axes is very small (for reference, $1000 \mathrm{~cm}^{-1}$ corresponds to approximately $124 \mathrm{meV}$ ). Indeed, the different structural domains found in our experiments for the $3 \mathrm{H}$ chain, as depicted in Figure 6a, are examples of the occurrence of such twists. On the other hand, the also very low frequencies of the longitudinal acoustic modes indicate that compressing or stretching the $3 \mathrm{H}$ chains also costs little energy. These characteristics are compatible with the nonmonotonic dependence reported here between encapsulating UNSWCNT diameter and encapsulated coil pitch: Since only small amounts of energy are needed for the Te $3 \mathrm{H}$ chains to be twisted, compressed, and stretched, the chains can easily adapt to the periodicity and chirality of the encapsulating UNSWCNTs.

\section{CONCLUSIONS}

We have reported the observation and modeling of extreme Te nanowires encapsulated inside UNSWCNTs with diameters between 0.7 and $1.1 \mathrm{~nm}$. Using state-of-the-art AC-TEM and AC-STEM methods, we have produced atomic-resolution images of such encapsulated extreme nanowires. Using the AIRSS method, we have successfully predicted the most energetically favorable structures formed by encapsulated Te. We have then produced a diagram of formation energy as a function of encapsulating diameters for such structures, providing thus a precise map of the diameter-dependent transitions undergone by the encapsulated extreme nanowires. Such transitions were shown to be electronic (metal-semiconductor) as well as structural. We found a remarkable agreement between our experimental observations and our theoretical modeling, especially with regards to the determination of the encapsulating nanotube diameters at which the structural transitions have been predicted to occur.

We analyzed the mechanical stability of the encapsulated Te extreme nanowires and determined whether or not they are expected to form. By doing so, we also demonstrated that nanoconfinement is not just a viable way to synthesize extreme nanowires. In many cases, it might actually be a necessary condition to prevent the nanowires from disintegrating spontaneously. In particular, we have shown that truly 1D, single-atom wide Te LCs can be synthesized provided that the confining diameters are small enough. Moreover, we established that such chains are longitudinally stabilized by the presence of Peierls structural distortions. We point out that the emergence of LCs is not an immediate consequence of extreme confinement. In fact, structures such as isolated atoms and dimers, for instance, are also geometrically compatible with the nanoconfimenent conditions to which our Te LCs are submitted and could thus have been detected/predicted if they were energetically favorable. Finally, for the Te three-fold-symmetry helical coil, we found that the pitch of the coil varies in a nonmonotonic fashion with the encapsulating diameters. Such an effect is related to the relatively low-energy cost involved in the processes of compressing, stretching, and twisting such coils, which allows them to adapt to the periodicity and chirality of the encapsulating nanotubes.

The excellent agreement between our theoretical modeling and the outcomes of our experiments shows that our experimental- 
theoretical approach provides a high-quality quantitative characterization of encapsulated extreme 1D nanowires, in fact, the most precise characterization of such systems presented to date. We thus anticipate that the present study will set the basic paradigm to be adopted in the characterization and understanding of nanoconfined 1D materials.

\section{EXPERIMENTAL METHODS}

Sample Preparation. The ENs are prepared by sublimation into pretreated SWeNT UNSWCNTs produced by the CoMoCAT process, ${ }^{41}$ supplied by Sigma-Aldrich with a stated median diameter range of $0.7-1.1 \mathrm{~nm} .100 \mathrm{mg}$ of UNSWCNTs were pretreated by heating in open air in an alumina boat placed in a Carbolite tube furnace (MTF $12 / 28 / 250$ ) to $750 \mathrm{~K}$ for $50 \mathrm{~min}$, reducing in mass to $60 \mathrm{mg} .100 \mathrm{mg}$ of Te (Sigma-Aldrich, $99.8 \%$ trace metals) was then loaded into one end of a sublimation ampule with the $60 \mathrm{mg}$ of UNSWCNT in the other centrally separated by an indentation in the center of the ampule. The ampule was sealed under vacuum and baked, with the Te section being placed in the center of the hot zone of the Carbolite tube furnace at $700 \mathrm{~K}$ for 6 days. Confirmation of sublimation arose from observations of Te crystallite deposition in the UNSWCNT region of the ampule by TEM. For TEM examination, 3 drops of dispersed CNTs were drop cast onto a $3.05 \mathrm{~mm}$ copper grid with lacey carbon support film (Agar Scientific).

HR-TEM and STEM-EELS. A JEOL ARM 200F microscope operating at $80 \mathrm{kV}$ and equipped with a CEOS aberration corrector and a Gatan SC1000 ORIUS camera with a $4008 \times 2672$ pixel CCD was been used for TEM investigations. Wien filtering was performed of selected TEM images using the script HRTEM Filter authored by D. R. G. Mitchell and based on the work of R. Kilaas. ${ }^{42}$ A Gatan fiberoptical coupled SC1000 ORIUS camera with CCD size of $4008 \times$ 2672 pixel was used for image acquisition. The STEM-EELS data were acquired on a Nion UltraSTEM100 instrument, operated at $60 \mathrm{kV}$ acceleration voltage and equipped with a cold field emission gun providing a native beam energy width of $0.30 \mathrm{eV}$. The combination of low acceleration voltage and ultrahigh vacuum (UHV) conditions at the sample (below $1 \times 10^{-9}$ Torr) ensured that damage to the samples, in particular through knock-on or etching, was minimal, enabling long beam dwell times for spectrum imaging. The optics were configured to form a $1.1 \AA$ probe (full-width at half-maximum) of $32 \mathrm{mrad}$ convergence semi-angle, with $40 \mathrm{pA}$ beam current. High- and mediumangle annular dark-field images were recorded with semi-angular ranges of $85-190$ and 55-80 mrad, respectively.

EELS data were collected with a Gatan Enfina spectrometer with a collection semi-angle of $36 \mathrm{mrad}$. A dispersion of $0.5 \mathrm{eV} /$ channel was chosen in order to record simultaneously the $\mathrm{C} \mathrm{K}$ and $\mathrm{Te} \mathrm{M}_{4,5}$ edges (resulting in an effective energy resolution of $1.5 \mathrm{eV}$, limited by the detector point spread function). Spectrum images were acquired with a dwell time of $0.05 \mathrm{~s}$ per pixel, providing a good compromise between signal-to-noise and possible damage to the Te wires within the nanotubes. The $\mathrm{Te}$ and $\mathrm{C}$ maps were generated by integrating the signal above the edge onsets over $75 \mathrm{eV}$ windows, after subtraction of the continuous background using a power law. Principal component analysis was carefully applied to the raw data sets to remove detector Poisson noise. ${ }^{43}$

\section{ASSOCIATED CONTENT}

\section{S Supporting Information}

The Supporting Information is available free of charge on the ACS Publications website at DOI: 10.1021/acsnano.7b02225.

More information about the structural models, adopted conventions, $^{44,45}$ terminology, and calculation ${ }^{46-53}$ parameters; detailed discussion about the ICNT model introduced here, as well as about our adaptation of AIRSS for 1D systems (including corrections of mismatchinduced unphysical strains); a general method to calculate the unstrained periodicity of encapsulated nanowires while fully accounting for the presence of the encapsulating (explicit) SWCNTs (PDF)

\section{AUTHOR INFORMATION}

\section{Corresponding Authors}

*E-mail: pvm20@cam.ac.uk; paulovcmedeiros@gmail.com.

*E-mail: j.sloan@warwick.ac.uk.

*E-mail: ajm255@cam.ac.uk.

ORCID $\odot$

Paulo V. C. Medeiros: 0000-0002-7803-9058

Andrij Vasylenko: 0000-0002-6933-0628

Notes

The authors declare no competing financial interest.

\section{ACKNOWLEDGMENTS}

P.V.C.M., A.J.M., A.V., and D.Q. thank the UK Engineering and Physical Sciences Research Council (EPSRC) for funding through grant numbers EP/M011925/1 and EP/M010643/1. J.M.W. acknowledges financial support from the EPSRC Centre for Doctoral Training in Computational Methods for Materials Science under grant EP/L015552/1. J.S. and S.M. are further indebted to support from EP/I033394/1. The SuperSTEM Laboratory is the U.K. National Facility for Aberration-Corrected STEM, supported by the EPSRC. This work used the ARCHER UK National Supercomputing Service (www.archer.ac.uk). Data used in this work are available via the Cambridge data repository at https://doi.org/10.17863/CAM.7096.

\section{REFERENCES}

(1) Spencer, J. H.; Nesbitt, J. M.; Trewhitt, H.; Kashtiban, R. J.; Bell, G.; Ivanov, V. G.; Faulques, E.; Sloan, J.; Smith, D. C. Raman Spectroscopy of Optical Transitions and Vibrational Energies of $1 \mathrm{~nm} \mathrm{HgTe}$ Extreme Nanowires within Single Walled Carbon Nanotubes. ACS Nano 2014, 8, 9044-9052.

(2) Sloan, J.; Wright, D. M.; Woo, H. G.; Bailey, S. R.; Brown, G.; York, A. P. E.; Coleman, K. S.; Hutchison, J. L.; Green, M. L. H. Capillarity and Silver Nanowire Formation Observed in Single Walled Carbon Nanotubes. Chem. Commun. 1999, 699-700.

(3) Massa, M. V.; Carvalho, J. L.; Dalnoki-Veress, K. Confinement Effects in Polymer Crystal Nucleation from the Bulk to Few-Chain Systems. Phys. Rev. Lett. 2006, 97, 247802.

(4) Carter, R.; Sloan, J.; Kirkland, A. I.; Meyer, R. R.; Lindan, P. J. D.; Lin, G.; Green, M. L. H.; Vlandas, A.; Hutchison, J. L.; Harding, J. Correlation of Structural and Electronic Properties in a New LowDimensional Form of Mercury Telluride. Phys. Rev. Lett. 2006, 96, 215501.

(5) Uemura, T.; Horike, S.; Kitagawa, K.; Mizuno, M.; Endo, K.; Bracco, S.; Comotti, A.; Sozzani, P.; Nagaoka, M.; Kitagawa, S. Conformation and Molecular Dynamics of Single Polystyrene Chain Confined in Coordination Nanospace. J. Am. Chem. Soc. 2008, 130, 6781-6788.

(6) Eliseev, A.; Yashina, L.; Kharlamova, M.; Kiselev, N. Electronic Properties of Carbon Nanotubes; InTech: Rijeka, Croatia, 2011; Chapter 8, pp 127-156.

(7) Ivanov, A. S.; Kar, T.; Boldyrev, A. I. Nanoscale Stabilization of Zintl Compounds: 1D Ionic Li-P Double Helix Confined inside a Carbon Nanotube. Nanoscale 2016, 8, 3454-3460.

(8) Vasylenko, A.; Wynn, J.; Medeiros, P. V. C.; Morris, A. J.; Sloan, J.; Quigley, D. Encapsulated Nanowires: Boosting Electronic Transport in Carbon Nanotubes. Phys. Rev. B: Condens. Matter Mater. Phys. 2017, 95, 121408.

(9) Desai, S. B.; Madhvapathy, S. R.; Sachid, A. B.; Llinas, J. P.; Wang, Q.; Ahn, G. H.; Pitner, G.; Kim, M. J.; Bokor, J.; Hu, C.; Wong, H.-S. P.; Javey, A. $\mathrm{MoS}_{2}$ Transistors with 1-Nanometer Gate Lengths. Science 2016, 354, 99-102.

(10) Gao, Y.; Bando, Y. Carbon Nanothermometer Containing Gallium. Nature 2002, 415, 599-599. 
(11) Winkler, A.; Mühl, T.; Menzel, S.; Kozhuharova-Koseva, R.; Hampel, S.; Leonhardt, A.; Büchner, B. Magnetic Force Microscopy Sensors Using Iron-Filled Carbon Nanotubes. J. Appl. Phys. (Melville, NY, U. S.) 2006, 99, 104905.

(12) Gautam, U. K.; Costa, P. M. F. J.; Bando, Y.; Fang, X.; Li, L.; Imura, M.; Golberg, D. Recent Developments in Inorganically Filled Carbon Nanotubes: Successes and Challenges. Sci. Technol. Adv. Mater. 2010, 11, 054501 .

(13) Hang, B. T.; Hayashi, H.; Yoon, S.-H.; Okada, S.; Yamaki, J.-i. $\mathrm{Fe}_{2} \mathrm{O}_{3}$-Filled Carbon Nanotubes as a Negative Electrode for an Fe-Air Battery. J. Power Sources 2008, 178, 393-401.

(14) Rossella, F.; Soldano, C.; Bellani, V.; Tommasini, M. Metal-Filled Carbon Nanotubes as a Novel Class of Photothermal Nanomaterials. Adv. Mater. (Weinheim, Ger.) 2012, 24, 2453-2458.

(15) Wuttig, M.; Yamada, N. Phase-Change Materials for Rewriteable Data Storage. Nat. Mater. 2007, 6, 824-832.

(16) Giusca, C. E.; Stolojan, V.; Sloan, J.; Börrnert, F.; Shiozawa, H.; Sader, K.; Rümmeli, M. H.; Büchner, B.; Silva, S. R. P. Confined Crystals of the Smallest Phase-Change Material. Nano Lett. 2013, 13, 40204027.

(17) Liu, Z.; Li, S.; Yang, Y.; Hu, Z.; Peng, S.; Liang, J.; Qian, Y. ShapeControlled Synthesis and Growth Mechanism of One-Dimensional Nanostructures of Trigonal Tellurium. New J. Chem. 2003, 27, 17481752 .

(18) Gautam, U. K.; Rao, C. N. R. Controlled Synthesis of Crystalline Tellurium Nanorods, Nanowires, Nanobelts and Related Structures by a Self-Seeding Solution Process. J. Mater. Chem. 2004, 14, 2530-2535.

(19) Liang, F.; Qian, H. Synthesis of Tellurium Nanowires and Their Transport Property. Mater. Chem. Phys. 2009, 113, 523-526.

(20) Kudryavtsev, A. A. The Chemistry and Technology of Selenium and Tellurium; Collet's Ltd.: London, 1974.

(21) Tsiulyanu, D.; Tsiulyanu, A.; Liess, H.-D.; Eisele, I. Characterization of Tellurium-Based Films for $\mathrm{NO}_{2}$ Detection. Thin Solid Films 2005, 485, 252-256.

(22) Miura, N.; Tanaka, S. Fast Photoconductivity of Tellurium by $\mathrm{CO}_{2}$ Laser Radiation. Appl. Phys. Lett. 1968, 12, 374-375.

(23) Liang, W.; Sai-Ling, H.; Fei, Z. Band Structures of TwoDimensional Photonic Crystals with Regular Polygon Cylinders Calculated by Linear Operations. Acta Phys. Sin. 2002, 51, 2865-2870.

(24) Kobayashi, K.; Yasuda, H. Structural Transition of Tellurium Encapsulated in Confined One-Dimensional Nanospaces Depending on the Diameter. Chem. Phys. Lett. 2015, 634, 60-65.

(25) Suenaga, K.; Sato, Y.; Liu, Z.; Kataura, H.; Okazaki, T.; Kimoto, K.; Sawada, H.; Sasaki, T.; Omoto, K.; Tomita, T.; Kaneyama, T.; Kondo, Y. Visualizing and Identifying Single Atoms Using Electron Energy-Loss Spectroscopy with Low Accelerating Voltage. Nat. Chem. 2009, 1, 415-418.

(26) Krivanek, O. L.; Chisholm, M. F.; Nicolosi, V.; Pennycook, T. J.; Corbin, G. J.; Dellby, N.; Murfitt, M. F.; Own, C. S.; Szilagyi, Z. S.; Oxley, M. P.; Pantelides, S. T.; Pennycook, S. J. Atom-by-Atom Structural and Chemical Analysis by Annular Dark-Field Electron Microscopy. Nature 2010, 464, 571-574.

(27) Pickard, C. J.; Needs, R. J. Ab Initio Random Structure Searching. J. Phys.: Condens. Matter 2011, 23, 053201.

(28) Morris, A. J.; Pickard, C. J.; Needs, R. J. Hydrogen/silicon Complexes in Silicon from Computational Searches. Phys. Rev. B: Condens. Matter Mater. Phys. 2008, 78, 184102.

(29) Morris, A. J.; Grey, C. P.; Needs, R. J.; Pickard, C. J. Energetics of Hydrogen/lithium Complexes in Silicon Analyzed Using the Maxwell Construction. Phys. Rev. B: Condens. Matter Mater. Phys. 2011, 84, 224106.

(30) Morris, A. J.; Needs, R. J.; Salager, E.; Grey, C. P.; Pickard, C. J. Lithiation of Silicon via Lithium Zintl-Defect Complexes from First Principles. Phys. Rev. B: Condens. Matter Mater. Phys. 2013, 87, 174108.

(31) Pickard, C. J.; Needs, R. J. Highly Compressed Ammonia Forms an Ionic Crystal. Nat. Mater. 2008, 7, 775-779.

(32) Ogata, K.; Salager, E.; Kerr, C.; Fraser, A.; Ducati, C.; Morris, A.; Hofmann, S.; Grey, C. Revealing Lithium-Silicide Phase Trans- formations in Nano-Structured Silicon-Based Lithium Ion Batteries via in Situ NMR Spectroscopy. Nat. Commun. 2014, 5, 3217.

(33) Senga, R.; Komsa, H.-P.; Liu, Z.; Hirose-Takai, K.; Krasheninnikov, A. V.; Suenaga, K. Atomic Structure and Dynamic Behaviour of Truly One-Dimensional Ionic Chains inside Carbon Nanotubes. Nat. Mater. 2014, 13, 1050-1054.

(34) Nicholls, R. J.; Morris, A. J.; Pickard, C. J.; Yates, J. R. OptaDOS - a New Tool for EELS Calculations. J. Phys.: Conf. Ser. 2012, 371, 012062.

(35) Pouget, J. P. The Peierls Instability and Charge Density Wave in One-Dimensional Electronic Conductors. C. R. Phys. 2016, 17, 332356.

(36) Batra, I. P. Gapless Peierls Transition. Phys. Rev. B: Condens. Matter Mater. Phys. 1990, 42, 9162-9165.

(37) Voit, J. Dynamical Correlation Functions of One-Dimensional Superconductors and Peierls and Mott Insulators. Eur. Phys. J. B 1998, 5, 505-519.

(38) Ghosh, P.; Kahaly, M.; Waghmare, U. Atomic and Electronic Structures, Elastic Properties, and Optical Conductivity of Bulk Te and te Nanowires: A First-Principles Study. Phys. Rev. B: Condens. Matter Mater. Phys. 2007, 75, 245437.

(39) Li, G.; Fu, C.; Oviedo, M. B.; Chen, M.; Tian, X.; Bekyarova, E.; Itkis, M. E.; Wong, B. M.; Guo, J.; Haddon, R. C. Giant Raman Response to the Encapsulation of Sulfur in Narrow Diameter Single-Walled Carbon Nanotubes. J. Am. Chem. Soc. 2016, 138, 40-43.

(40) Gómez-Rodríguez, A.; Beltrán-del Río, L.; Herrera-Becerra, R. SimulaTEM: Multislice Simulations for General Objects. Ultramicroscopy 2010, 110, 95-104.

(41) Jorio, A.; Santos, A. P.; Ribeiro, H. B.; Fantini, C.; Souza, M.; Vieira, J. P. M.; Furtado, C. A.; Jiang, J.; Saito, R.; Balzano, L.; Resasco, D. E.; Pimenta, M. A. Quantifying Carbon-Nanotube Species with Resonance Raman Scattering. Phys. Rev. B: Condens. Matter Mater. Phys. 2005, 72, 075207.

(42) Kilaas, R. Optimal and Near-Optimal Filters in High-Resolution Electron Microscopy. J. Microsc. (Oxford, U. K.) 1998, 190, 45-51.

(43) Watanabe, M.; Okunishi, E.; Ishizuka, K. Analysis of SpectrumImaging Datasets in Atomic-Resolution Electron Microscopy. Microsc. Anal. 2009, 23, 5-7.

(44) Saito, R.; Dresselhaus, G.; Dresselhaus, M. S. Physical Properties of Carbon Nanotubes; Imperial College Press: London, 2001.

(45) Qin, L.-C. Determination of the Chiral Indices $(n, m)$ of Carbon Nanotubes by Electron Diffraction. Phys. Chem. Chem. Phys. 2007, 9, $31-48$.

(46) Hohenberg, P.; Kohn, W. Inhomogeneous Electron Gas. Phys. Rev. 1964, 136, B864.

(47) Kohn, W.; Sham, L. J. Self-Consistent Equations Including Exchange and Correlation Effects. Phys. Rev. 1965, 140, A1133.

(48) Clark, S. J.; Segall, M. D.; Pickard, C. J.; Hasnip, P. J.; Probert, M. I. J.; Refson, K.; Payne, M. C. First Principles Methods Using CASTEP. Z. Kristallogr. - Cryst. Mater. 2005, 220, 567-570.

(49) Vanderbilt, D. Soft Self-Consistent Pseudopotentials in a Generalized Eigenvalue Formalism. Phys. Rev. B: Condens. Matter Mater. Phys. 1990, 41, 7892-7895.

(50) Kozinsky, B.; Marzari, N. Static Dielectric Properties of Carbon Nanotubes from First Principles. Phys. Rev. Lett. 2006, 96, 166801.

(51) Arfken, G. B., Weber, H. J. Mathematical Methods for Physicists, 6th ed.; Academic Press: San Diego, 2005.

(52) VanSiclen, C. Estimating Atomic 1s Orbital Radii. J. Comput. Appl. Math. 1987, 19, 283-286.

(53) Vinet, P.; Smith, J. R.; Ferrante, J.; Rose, J. H. Temperature Effects on the Universal Equation of State of Solids. Phys. Rev. B: Condens. Matter Mater. Phys. 1987, 35, 1945-1953. 